\title{
Engineering social contagions: Optimal network seeding in the presence of homophily
}

\author{
SINAN ARAL \\ MIT Sloan School of Management, 100 Main Street, Cambridge, MA 02139, USA \\ (e-mail: sinana@mit.edu) \\ LEV MUCHNIK \\ Hebrew University of Jerusalem School of Business Administration, 5102b, Jerusalem, Israel 91905 \\ (e-mail: lev.muchnik@huji.ac.il) \\ ARUN SUNDARARAJAN \\ NYU Stern School of Business, 44 West 4th Street Room 8-93, New York, NY 10012, USA \\ (e-mail: arun@stern.nyu.edu)
}

\begin{abstract}
We use data on a real, large-scale social network of 27 million individuals interacting daily, together with the day-by-day adoption of a new mobile service product, to inform, build, and analyze data-driven simulations of the effectiveness of seeding (network targeting) strategies under different social conditions. Three main results emerge from our simulations. First, failure to consider homophily creates significant overestimation of the effectiveness of seeding strategies, casting doubt on conclusions drawn by simulation studies that do not model homophily. Second, seeding is constrained by the small fraction of potential influencers that exist in the network. We find that seeding more than $0.2 \%$ of the population is wasteful because the gain from their adoption is lower than the gain from their natural adoption (without seeding). Third, seeding is more effective in the presence of greater social influence. Stronger peer influence creates a greater than additive effect when combined with seeding. Our findings call into question some conventional wisdom about these strategies and suggest that their overall effectiveness may be overestimated.
\end{abstract}

Keywords: peer influence, social contagion, social networks, viral marketing, information systems, simulation

\section{Introduction}

Firms and policymakers are increasingly recruiting customers to take part in wordof-mouth (WOM) marketing campaigns. Seeding or network targeting has emerged as the primary marketing strategy designed to leverage the WOM effect (Domingos \& Richardson, 2001; Lazarsfeld et al., 1948; Valente \& Davis, 1999) (for an excellent review of Network Interventions, see Valente, 2012). Seeding seeks to convert influential individuals who are expected to use their social network position, personal influence, and broad peer contacts to trigger cascades of product adoption. A variety of different seeding strategies have been proposed, such as those that target hubs or dense regions of the network, and many programs are beginning to 
harness the power of these strategies. ${ }^{1}$ However, little is known about their relative effectiveness. In particular, we know little about how different seeding strategies perform under different social conditions. For example, different levels of peer influence or homophily in a diffusion process may affect the relative performance of different network seeding policies.

Today's state-of-the-art viral marketing simulations typically consider social network structures derived from random or small world graphs rather than real networks, and do not incorporate empirically observed distributions of adoption propensities, correlations between individuals' personal and network characteristics, or similarities among connected network neighbors (homophily) (Abrahamson \& Rosenkopf, 1997; Centola \& Macy, 2007; Goldenberg et al., 2001; Kempe et al., 2003; Kitsak et al., 2010; Liben-Nowell \& Kleinberg, 2008; Van den Bulte \& Joshi, 2007). All of these factors are known to have profound impacts on diffusion dynamics and the causal mechanisms driving adoption (Aral et al., 2009). Unconstrained by data, different modeling assumptions generate differing conclusions, creating disagreement about the efficacy of different seeding strategies (e.g. Watts and Dodds, 2007; Gladwell, 2000).

Empirical work on the other hand, which estimates WOM using observational data or randomized experiments, is more realistic but rarely analyzes global diffusion outcomes or multiple competing intervention strategies (Aral et al., 2009; Aral \& Van Alstyne, 2011; Aral \& Walker, 2011; Bakshy et al., 2012a; Bakshy et al., 2012b; Centola, 2010, Centola, 2011; Eagle et al., 2010; Leider et al., 2009; Leskovec et al., 2007; Sun et al., 2009; Taylor et al., 2013). Single marketing actions are assessed in isolation to maintain precision and experimental control and typically only immediate neighbor effects are estimated. As a result, most empirical studies can only make bounded generalizations about the global effects of seeding.

In this paper, we complement and extend current work on seeding strategies by combining the flexible but abstract simulation-based approach with the more realistic but limited empirical approach. We use data on a real, large-scale social network of 27 million individuals interacting daily, together with detailed observations of the same individuals' demographic and personal characteristics and online browsing behaviors, and their day-by-day adoption of a new mobile service product, to inform, build, and analyze data-driven simulations of the effectiveness of WOM marketing strategies under different social conditions. We employ this framework to explore "what if" scenarios representing a series of seeding strategies under different assumptions about the level of influence and homophily in the system. Data-driven simulations are a useful way to gauge the likely effects of firm policy. However, few data-driven simulations of networked interventions exist and none examine seeding under varying levels of homophily and influence.

Three main results emerge from our simulations. First, failure to consider homophily creates significant overestimation of the effectiveness of different seeding strategies. We find that systems with empirically observed amounts of homophily experience $30 \%$ less adoption from WOM than identical systems without homophily,

1 One mainstream example is SponsoredTweets, which connects firms to influential users of Twitter.com so they can be paid to tweet about products (see http://sponsoredtweets.com/). Another example is the Klout Perks program, which gives free products to people assessed as "influential." 
casting doubt on conclusions drawn by simulation studies that do not model homophily.

Second, seeding is constrained by the small fraction of potential influencers that exist in the network. We find that even when the most influential individuals are targeted, seeding more than $0.2 \%$ of the population is wasteful because the gain from their adoption, in adoptions triggered by their conversion, is lower than the gain from their natural adoption (without seeding).

Third, seeding is more effective in the presence of greater social influence. Stronger influence creates a greater than additive effect when combined with seeding. Though the complementary effect is relatively small, the interaction between influence and seeding is complex. High degree individuals are more likely to be early adopters in systems with significant peer influence because their degree exposes them to natural influence early on in the diffusion process. This effect creates diminishing marginal complementarities between seeding and influence as influence increases. We investigate these effects and discuss their implications for marketing and networked interventions.

Our findings call into question some of the conventional wisdom about the most popular viral marketing strategies and suggest that their overall effectiveness may be overestimated. Given the increasing availability of relevant data (Lazer et al., 2009) empirically motivated simulations such as ours could contribute greatly to our understanding of network marketing in the near future. ${ }^{2}$

\section{Model design}

\subsection{Data}

We began our investigation by collecting a longitudinal dataset that combines (i) the global network of daily instant messaging (IM) traffic between 27.4 million users of Yahoo.com with (ii) data on the day-by-day adoption of a mobile service application launched in July 2007 (Yahoo Go), ${ }^{3}$ and (iii) robust and precise attribute and dynamic behavioral data on users' demographics, geographic location, mobile device type and usage, and per-day page views of different types of content (e.g. sports, weather, news, finance, and photo sharing) from desktop, mobile, and Go platforms. Much of these data, such as mobile device usage and page views of different types of content, provide fine-grained proxies for individuals' tastes and preferences. The complete set of covariates includes 40 time-varying and 6 timeinvariant individual and network characteristics. Taken together, the sampled users of the IM network registered over 14 billion page views and sent 3.9 billion messages over 89.3 million distinct relationships (for details about the service, the data, and descriptive statistics, see the SOM and (Aral et al., 2009).

2 We have made the data publicly available directly through Yahoo!'s Webscope Program to encourage validation of our results and new empirical research on network intervention (http://webscope.sandbox.yahoo.com/). We intend to make the code for our simulations publicly available as well. Note we are legally prohibited from releasing the data to anyone ourselves. All data inquiries should be addressed directly to Yahoo! through their Webscope Program.

3 The Yahoo Go mobile service application we studied is described in more detail in the Supplementary Online Material (SOM). 
The data represent an anonymized sample of the Yahoo! IM network, where each node is an IM user for whom we collected detailed demographic, geographic and behavioral information as well as daily IM message traffic. We first sampled all Yahoo! IM users who adopted Yahoo! Go between June 1, 2007 and October 29, 2007. This "seed experimental sample" consists of 532,365 users that we labeled "service adopters." We then created a "seed control sample" by taking a random sample of $2 \%$ of the entire IM network. This "seed control sample" consists of 2,974,288 nodes that we labeled "random control seeds." We executed a two-step snowball sampling procedure which traversed network links, defined by the existence of IM message traffic, two steps out from every control and experimental seed node, collecting the complete local network neighborhoods of all seed nodes. The first step of the snowball sampling procedure yielded 9.1 million new nodes that were IM contacts of the seed node populations ("first-step nodes"). We then collected the local networks of all first-step nodes by sampling all users who received at least one message from them. The second step of the snowball sampling procedure yielded an additional 14.9 million users two steps away from a seed node.

\subsection{Modeling product adoption}

Recent analytical models of peer influence that consider network structure (Galeotti \& Goyal, 2009; Goyal et al., 2010; Sundararajan, 2008) are better able to explain observed behavioral diffusion patterns than early models that do not consider interpersonal interaction (Chatfield \& Goodhardt, 1973; Uncles et al., 1995) or the network structure of those interactions (Bass, 1969). However, more sophisticated models that account for realistic, empirically observed network structures, and distributions of individual characteristics over nodes have not yet been developed.

We used the data described in the prior section to develop and train such a model of product adoption. We define a time-varying, individual-specific adoption propensity measure for each consumer. This is akin to an adoption probability or perceived value; the higher this measure, the more likely a consumer is to adopt the product. This propensity is a function of two concurrent factors that drive adoption: characteristic-based preferences and peer influence. This partition is broadly motivated by the widely used Bass diffusion model (Bass, 1969), which describes the process of new product adoption as a population-level interaction between users and potential users that is governed by an innovation rate driving product adoption by the population independent of installed base, and an imitation rate that drives an increase in product adoption as a linear function of the installed base. Correspondingly, in our formulation, characteristic-based adoption describes a consumer's propensity to adopt the product as a function of their individual characteristics. Peer influence then incorporates the influence of an individual's peers on their adoption propensity. The overall propensity of a user to adopt the product is the sum of their propensity to adopt as determined by their characteristics, and their propensity to adopt as determined by their peers:

$$
P_{i t}^{A}=P_{i t}^{C H}+P_{i t}^{I},
$$

where $P_{i t}^{A}$ is the overall propensity that user $i$ will adopt the product at time $t, P_{i t}^{C H}$ is the propensity that user $i$ will adopt the product at time $t$ due to their individual 
characteristics, and $P_{i t}^{I}$, the propensity that user $i$ will adopt the product at time $t$ owing to the aggregated peer influence of $i$ 's immediate neighbors at time $t$. The linear composition of the probabilities does not necessary imply independence of $P_{i t}^{C H}$ and $P_{i t}^{I}$ since, as we consider later, susceptibility to peer influence may depend on the propensity to adopt spontaneously due to individual characteristics (Schmitt et al., 2011).

We use logistic regression to estimate the characteristic-based propensity of an individual to adopt the product at time $t$ as a function of their own characteristics:

$$
P_{i t}^{C H}=\frac{\exp (z)}{1+\exp (z)}, \quad z=\beta_{0}+\sum_{k} \beta_{k} x_{i k t}+\varepsilon_{i t},
$$

where $x_{i t}$ is a vector of 40 time-varying and 6 time-invariant user characteristics that contains personal demographics characteristics, behavioral attributes such as browsing behavior (a proxy for personal preferences for web-based content that varies by subject and theme), local network measures, averages of users' neighbors characteristics, and distance from a seed node (see SOM for a full description of the estimation strategy and results).

We then model peer influence as diffusing through the structure of observed IM communication. Given the similarity between the product facilitating IM communication and the product being adopted (Yahoo Go) (both require authentication with the same firm and complement each other), we assume that the IM network closely resembles the underlying social network conveying WOM about the product. This assumption is supported by the strong correlations in product adoption and observable characteristics among connected peers and that IM communication and individual characteristics are good predictors of product adoption (Aral et al., 2009).

Specifically, $i$ 's propensity to adopt due to peer influence is given by:

$$
P_{i t}^{I}=\sum_{j \in N_{i}} w_{i j t} y_{j t-1} I_{j i t},
$$

where $N_{i}$ is the set of network neighbors $j$ of node $i, w_{i j t}$ is the tie strength of $i$ and $j$ at time $t$, measured by the number of instant messages exchanged between them, $y_{j t-1}$ is the adoption status of $j$ at time $t-1$ (where $y_{j t-1}=1$ if $j$ has adopted the product in the previous period $t-1$ and is 0 otherwise), and $I_{j i t}$ is an influence factor that describes the magnitude of $j$ 's influence on $i$ 's adoption decision at time $t$. One might think of the product of $w_{i j t}$ and $I_{j i t}$ as being a time-varying, dyad-specific version of the static, population-level imitation rate of the Bass diffusion model.

We assume that a potential adopter may only be influenced by prior adopters (when $y_{j t-1}=0$, the influence term goes to zero for that peer). While this assumption is not conceptually necessary and can be relaxed without loss of generality, it is quite reasonable. We make product adoption a prerequisite for peer influence because observational learning is unlikely unless a peer has adopted the product. Prior experience with the product is likely correlated with an adopter's desire to discuss the product with friends as well as their friends' likelihood of finding such discussions persuasive. This restriction is common in social contagion research (Aral, 2011; Iyengar et al., 2011) and most studies find that local social environments encourage a behavior only after that behavior becomes prevalent in the environment (Whyte, 1954). In addition, behavioral correlations in networks are typically short-ranged 
and do not exceed two hops (Christakis \& Fowler, 2007), meaning the likelihood of the friend of a friend of an adopter being influenced by the original adopter's decision (without the friend adopting) is low. The precise functional form of peer influence $I_{j i t}$, the contribution of adopter friend $j$ to $i$ 's propensity to adopt at time $t$, is the subject of different modeling assumptions throughout the paper, as described in detail in the rest of this section.

We convert the adoption propensity into a single composite probability of adoption per user per time period. It is important to note that in our simulations we use this as a "single trial" probability of adoption. An alternative, which follows the approach used in epidemiological models (Barthélemy et al., 2004; Pastor-Satorras \& Vespignani, 2001), might instead assume that each peer generates an independent adoption trial with probability $w_{i j t} y_{j t-1} I_{i j t}$, and if consumer $i$ has $k$ adopting peers at time $t$, their adoption outcome is thus determined by $k+1$ independent trials. This alternative represents an interesting direction for future research.

Our different functional forms for peer influence are motivated by a common belief that firms can affect the peer influence mechanisms at work in a population (Buttle, 1998) and that peer influence may naturally vary depending on the characteristics of the product and the social environment in which adoption is occurring (Aral \& Walker, 2011). Specifically, a firm may encourage current adopters to exert more influence on their peers (Godes \& Mayzlin, 2009) or alternatively may increase the susceptibility of potential customers to the influence of their peers (Aral \& Taylor, 2011); some firms may choose to do both. We therefore isolate and test two families of plausible peer influence mechanisms in realistic product diffusion scenarios - one which assumes that influence is driven by the persuasion of adopters' and another which assumes that influence is instead driven by the susceptibility to influence of adopters' peers (Aral \& Walker, 2012). These two influence mechanisms capture the ongoing debate about whether influentials or masses of susceptible consumers drive contagion (Gladwell, 2000; Katz \& Lazarsfeld, 1955; Watts \& Dodds, 2007).

As a baseline, we assume a persuasion-driven peer influence mechanism (Buttle, 1998) frequently used by researchers to model the diffusion of product adoption (Biyalogorsky et al., 2008; Buttle, 1998). The persuasion model assumes that a user's excitement about the product is proportional to their intrinsic likelihood of adoption as a function of their own characteristics and preferences $P_{i t}^{C H}$. The tendency to adopt the product due to one's own characteristics and preferences is correlated with user satisfaction, which is in turn correlated with the likelihood of engaging in positive WOM and thus persuasion. In other words, those who prefer the product more are more likely to talk about it.

We also incorporate a second influence mechanism which assumes that the susceptibility to influence is proportional to one's intrinsic likelihood of product adoption as a function of one's own characteristics and preferences. This approach assumes that those who are likely to be interested in the product are more likely to listen to and be persuaded by positive WOM. Thus, the influence term is a weighted sum of one's friends' and one's own likelihood of adopting the product due to personal characteristics and preferences, scaled by $\gamma$. As $\gamma$ increases, the persuasion of an adopter (which is proportional to their adoption likelihood) increases. As $\gamma$ decreases, the susceptibility to peer influence of adopters' friends 
(which is proportional to their adoption likelihood) increases:

$$
I_{j i t}=\kappa\left[(1-\gamma) \cdot\left(P_{j t}^{C H}\right)+\gamma \cdot\left(P_{i t}^{C H}\right)\right] .
$$

The overall amount of influence in the system is then scaled by $\kappa$, the magnitude of the effect of peer influence on the likelihood of adoption. Thus, the influence of $j$ on $i$ at time $t$ is given by:

$$
P_{i t}^{I}=\kappa \sum_{j \in N_{i}} w_{i j t} y_{j t-1}\left[(1-\gamma) \cdot\left(P_{j t}^{C H}\right)+\gamma \cdot\left(P_{i t}^{C H}\right)\right] .
$$

In order to establish the effect of seeding under different social conditions, we must first empirically estimate the amount of observed peer influence in the system. In general, a firm can estimate natural peer influence through a series of controlled randomized experiments designed to separate influence from homophily and characteristics-based adoption (Aral \& Walker, 2011; Aral \& Walker, 2012; Centola, 2010, Centola, 2011). Alternatively, methods such as dynamic propensity score matching can estimate the amount of natural peer influence in the system (Aral et al., 2009). To separate influence from homophily and other confounds, we created a dynamic matched sample of "treated" and "untreated" nodes over time, where receiving varying degrees of the "treatment" is defined as having 1, 2, 3 , 4, or more friends who adopted the product. We matched treated nodes with untreated nodes that were as likely to have the same number of adopter friends, conditional on a vector of observable characteristics and behaviors, but who did not have as many adopter friends. Conditioning matches on a vector of observable characteristics, behaviors, and attributes yields influence estimates that account for the homophily that may make product adoption decisions cluster in the network even if no influence exists (for complete details of the procedure, see SOM and Aral et al. (2009)).

Combining estimates of characteristics-based adoption, estimated using propensity score matching, with peer influence yields the following general expression for the propensity of user $i$ to adopt the product at time $t$ :

$$
\begin{aligned}
P_{i t}^{A}(x, w, y, \kappa, \gamma)= & \left(\frac{\exp \left(\beta_{0}+\sum_{k} \beta_{k} x_{i k t}\right)}{1+\exp \left(\beta_{0}+\sum_{k} \beta_{k} x_{i k t}\right)}\right) \\
& +\kappa \sum_{j \in N_{i}} w_{i j t} y_{j t-1}\left[(1-\gamma) \cdot\left(P_{j t}^{C H}\right)+\gamma \cdot\left(P_{i t}^{C H}\right)\right] .
\end{aligned}
$$

The probability of $i$ 's adoption at time $t$ can then be generated by an appropriate rescaling of the adoption propensity $P_{i t}^{A}(x, w, y, \kappa, \gamma)$. Given the manner in which we estimate $P_{j t}^{C H}$ to begin with using Equation (2), as an actual estimated probability of adoption from our data, we rescale the propensity measure by asserting a cutoff: the probability of adoption is $\max \left\{1, P_{i t}^{A}(x, w, y, \kappa, \gamma)\right\}$. This places an upper bound on the extent to which one's peers might increase one's propensity to adopt. Relative to the natural alternative of proportional normalization, this approach preserves the information inferred from our network data more effectively. It also combines aspects of a threshold model with those of a probabilistic model-we are asserting that if an individual's propensity to adopt is greater than a threshold, they will 
adopt with certainty, and if it is lower than this threshold, they will adopt with some probability less than one.

In the next several sections, we describe our models of seeding. By varying $\gamma$ from 0 to 1 , we examine the effects of seeding under varying assumptions about the degree to which influence or susceptibility to influence drives product adoption. We vary the degree to which adopters promoting the product to peers $(\gamma=0)$ or alternatively the susceptibility of potential adopters to peer influence $(\gamma=1)$ drive product adoption. Modeling persuasion and susceptibility in this way allows us to test their relative effects on seeding but does not necessarily imply that the two processes tradeoff. We model simultaneous increases in susceptibility and persuasion by increasing the overall level of influence $(\kappa)$. We conclude with an investigation of the effectiveness of seeding under varying assumptions about the level of influence in the system and whether that influence operates primarily through the persuasion of adopting peers $(\gamma=0)$ or the susceptibility of friends of adopters to peer influence $(\gamma=1)$.

\subsection{Modeling network seeding strategies}

Katz and Lazarsfeld (1955) suggested that the opinions of the majority may be shaped by a small subset of influential individuals who filter and reinterpret the mass media. Since then, research has actively investigated seeding strategies that attempt to identify and convert these influential individuals (Galeotti \& Goyal, 2009; Goldenberg et al., 2009; Iyengar et al., 2011; Marsden, 2006; Rosen, 2009; Valente \& Davis, 1999; Van den Bulte \& Joshi, 2007; Watts \& Dodds, 2007). Some argue that opinion leaders typically have high degree and occupy central positions in social networks (Becker, 1970). In recent years, as large-scale network data became available and typical network structures were explored (Barabási, 2002; Newman, 2003), the view that central hubs with high degree were the most influential in generating information cascades received substantial support from several domains (Cohen et al., 2001; Dezső \& Barabási, 2002; Pastor-Satorras \& Vespignani, 2001). A frequent approach in simulation models of WOM marketing ties individuals' degree to the amount of influence they are likely to generate (Libai et al., 2009).

We test a number of such seeding strategies and assess their effect on contagion in a realistic population. In devising seeding strategies, firms rank users according to a set of assumptions linking personal or network attributes to the ability to generate WOM influence and then designate how many individuals to target. The firm would then target the selected users to receive the product, for example in an email with a download link, in effect seeding their adoption. We explore each seeding strategy by varying assumptions about influence along dimensions proposed in the literature and by manipulating the fraction of the population that is targeted. We compare the simulated results of each strategy to the baseline simulation of no seeding which relies on the observed natural product diffusion dynamics of Yahoo Go.

Random seeding. We start with a naïve approach that targets random individuals. Firms occasionally launch marketing campaigns that, to a first approximation, are equally likely to reach every person in the population. This approach is easy to implement and does not require any knowledge of the underlying network. By seeding randomly, the firm expects to occasionally hit an influential individual whose WOM contribution will justify the advertising investment by triggering a substantial 
adoption cascade. While we do not expect this approach to yield impressive results, we recognize its value as a baseline case. Other researchers have also used the performance of random seeding as a baseline against which to evaluate more efficient strategies (Libai et al., 2009). To model this strategy, we simply rank nodes randomly and choose a fraction of the population to target.

Highest degree. The second approach assumes that the number of adoptions induced by an individual is correlated with their network degree. More people are likely to be exposed to peer influence from high-degree adopters. Some research supports the claim that high network degree is a reliable indicator of ability to influence (Libai et al., 2009). Given that typical social networks are characterized by highly skewed degree distributions (Barabási, 2002; Newman, 2003), it is not surprising that a significant portion of the population are in contact with one of the hubs and can be exposed to influence by their conversion. Individuals with many friends are thought to be particularly gregarious, active, and persuasive (Marsden, 2006; Rosen, 2009). Those with the personality to be opinion leaders also tend to assume central network positions (Freeman, 1979; Valente \& Davis, 1999).

The role of highly connected individuals has been tested in a large number of networks ranging from sexual contact networks used to model the spread of diseases (Barthélemy et al., 2004; Pastor-Satorras \& Vespignani, 2001), to the Internet (Cohen et al., 2001), to terrorist networks (Krebs, 2002). A number of models also test the role of hubs in the diffusion of new products (Goldenberg et al., 2009). The increasing interest in hubs as ideal targets in network marketing is justified by their potential to influence a significant fraction of the population and because finding them is cheap. As hubs can be found using local network measures such as degree, firms do not need to collect complete network data or detailed personal characteristics. A sample of user degrees is relatively easy to obtain. For example, even random walk sampling is biased toward highly connected nodes and can be used to effectively collect information about hubs (Borgatti, 2005; Rosvall \& Bergstrom, 2008). To model this strategy, we simply rank nodes by their degree and choose a fraction of the population to target.

Dense regions. An arguably more efficient way to reach more people is to target those who can reach the maximum number of users in two steps. This approach avoids seeding high degree nodes in unconnected components or sparsely populated areas of the graph and ensures that targets are located in the core of the network rather than on its periphery. Kitsak et al. (2010) find that users connected to a large number of well-connected individuals may have a particularly significant role in information diffusion. They simulate the diffusion of a contagious disease in a number of social networks and find that the most efficient spreaders are the users in the innermost $k$-core, rather than simply those connected to a large number of peers. To test this strategy, we rank nodes by the maximum number of unique nodes they can reach in two hops and choose a fraction of the population to target.

Hubs unlikely to adopt. Another strategy targets hubs that are unlikely to adopt on their own (i.e. nodes exhibiting relatively high degree and $\operatorname{low} P^{C H}$ ). Evidence suggests that highly connected influentials are themselves more likely to be exposed to outside influence and to adopt naturally (Becker, 1970). Hubs may also adopt earlier simply because they are more likely to be exposed to the product due to their high degree (Dover et al., 2009; Goldenberg et al., 2009). However, a large fraction 
of product adoption in our data is driven by spontaneous characteristics-based adoption and not by peer influence. Furthermore, hubs that are unlikely to adopt as a function of their own characteristics tend to be surrounded by users that are also unlikely to adopt because networks are homophilous. This further reduces the likelihood that this type of hub will adopt through contagion. On the other hand, seeding such individuals may affect a larger number of users that would otherwise not be exposed to the product because these hubs are located in areas of the network that are less likely to adopt. Whether this strategy works in practice is therefore an interesting question which we attempt to answer through simulation.

Formally, we compute this rank by assigning a score $S_{i}$ to each node which represents their fitness to the criteria described:

$$
S_{i}=\log \left(R_{i}^{d}\right)+\log \left(R_{i}^{P^{C H}}\right)
$$

Here, $R_{i}^{d}$ is the rank of the individual in descending degree order (e.g. $R_{i}^{d}=1$ is the individual with the highest degree) and $R_{i}^{p^{C H}}$ is the rank of the individual ordered by their estimated propensity to adopt spontaneously due to their individual characteristics $\left(R_{i}^{P^{C H}}=1\right.$ represents the individual with the lowest $P_{i}^{C H}$ in the network). Once the score is computed, the nodes are ranked in ascending order. The ranks are $\log$ transformed because degree and $P_{i}^{C H}$ distributions are highly skewed and roughly power law.

\subsection{Modeling variation in influence}

To model the effects of variation in influence $\left(I_{j i t}\right)$, we allow the parameters $\kappa$ and $\gamma$ to vary and evaluate the effects of such variation on product diffusion and the effectiveness of seeding. The influence of WOM generated by customers can vary as a function of the characteristics of the product (e.g. the product's popularity or the degree to which the product exhibits network externalities). In addition, the introduction of referral programs may stimulate adopters to convince their friends to adopt (Libai et al., 2003). Referral programs provide monetary incentives to adopters who encourage their friends to adopt and to potential adopters who are referred by a friend. For example, the firm could offer a discount to consumers for every friend they refer (Biyalogorsky et al., 2008; Buttle, 1998; Libai et al., 2003; Reingen \& Kernan, 1986; Schmitt et al., 2011). Alternatively, the firm could offer an incentive to every new adopter who was referred by a friend (Aral \& Taylor, 2011). Rather than encouraging persuasion by prior adopters, the program amplifies the susceptibility of potential adopters to peer influence. As a result of variation in product characteristics or firm incentive policies, influence can be driven by the propensity for adopters to engage in $\operatorname{WOM}(\gamma=0)$ or the propensity for friends of adopters to be susceptible to peer influence generated by WOM $(\gamma=1)$. Persuasion and susceptibility can also grow simultaneously: $\kappa$ is assumed to grow with a natural increase in peer influence or additional investments in marketing initiatives aimed at generating positive WOM and peers' susceptibility to positive WOM (Biyalogorsky et al., 2008). 


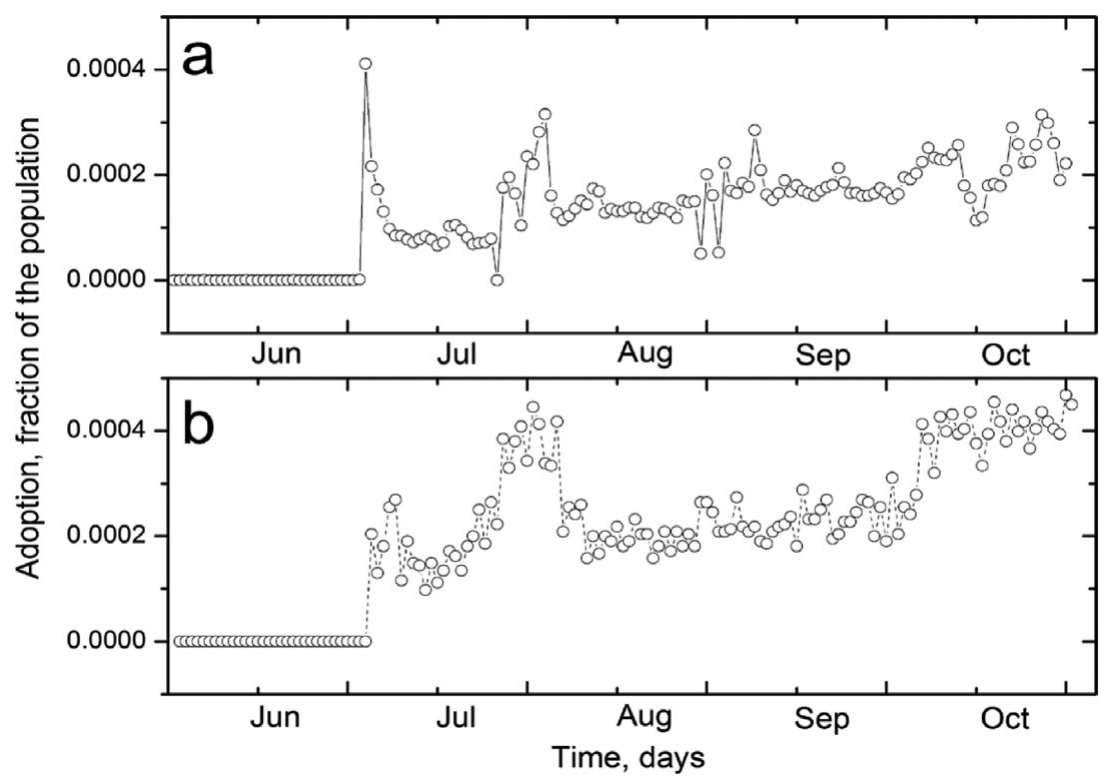

Fig. 1. Graphs of the adoption curve in the first five months since product launch. The observed (a) and the reconstructed (b) fractions of the daily adopters in the entire population are shown.

\section{Results}

\subsection{Baseline results}

When the dynamic matched sampling technique is applied to our data, we find that at least $50 \%$ of Yahoo Go adoption can be explained by spontaneous characteristicsbased adoption (Aral et al., 2009). This estimate corresponds to $\kappa \sim 0.50$ for the interval in question. In other words, the amount of peer influence generated with the excitement model $(\gamma=0)$ that approximates influence present in the observed data is obtained when half of adopter peers' own estimated propensity to adopt due to individual characteristics $\left(P_{j t}^{C H}\right)$ is transferred as influence on each of her neighbors.

Using the baseline model described in Equation (6), data on the adoption of Yahoo GO in the IM network and individuals' personal characteristics and browsing behaviors, we empirically estimated the propensity of product adoption of each user in the Yahoo IM network over time $\left(P_{i t}^{A}\right)$ as well as the likelihood of characteristicbased adoption $\left(P_{i t}^{C H}\right)$ and influence-based adoption $\left(P_{i t}^{I}\right)$. Figure 1 demonstrates that our baseline model produces an accurate and precise reconstruction of the daily adoption curve observed in the empirical data, giving us confidence in our modeling approach.

The first simulation provides a baseline model against which all seeding simulations are compared. The baseline simulations produce a distribution of spontaneous adoption probabilities, displayed in Figure 2(a), which is skewed and heavy-tailed. Most users have a low probability of adoption, but some have up to a $45 \%$ chance of adopting without firm intervention. Figure 2(b) displays the simulated number of adopters per day when only characteristics-based adoption is considered and peer-to-peer influence is set to zero (Spontaneous Adoption: $\kappa=0$ ), as well as when a natural level of organic peer influence through WOM (as empirically estimated 

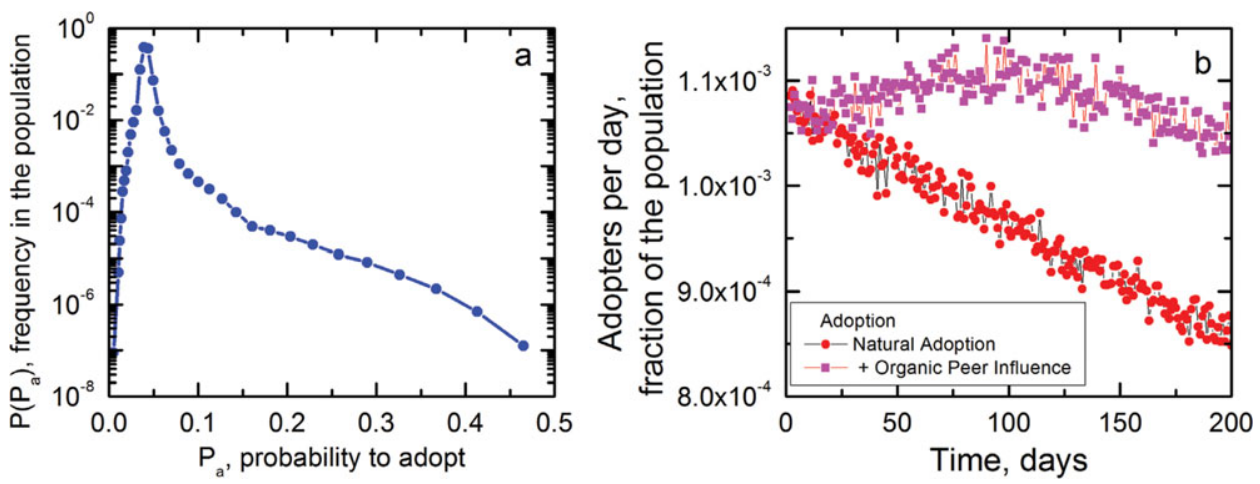

Fig. 2. (a) The distribution of spontaneous product adoption probabilities and (b) the simulated number of adopters per day under natural adoption and natural adoption + organic peer influence, $\kappa=0.5$. The plot represents persuasion-driven peer influence mechanism defined by $\gamma=0$. (color online)

in the data) is included in the simulation by setting $\kappa \sim 0.5$ (natural adoption + estimated organic peer influence).

Without peer influence, the number of adopters per day declines steadily over time (see Figure 2(b)). Users with the greatest propensity to adopt do so early on. Over time the number of likely adopters in the population is exhausted, creating declining numbers of adopters per day. When organic influence is considered, initial adopters influence their friends and thus increase their probability of adoption, increasing the number of adopters per day for approximately the first 100 days of the simulation, after which saturation again sets in and those who are going to adopt do so until available adopters are exhausted in the network. Two important insights emerge from the baseline simulation: (1) peer influence increases the likelihood that peers of adopters will adopt the product, boosting total adoption in the network, and (2) adoption decreases over time as the consumer population becomes saturated. In what follows, we alter this baseline model to simulate various firm interventions and compare outcomes to evaluate the relative effectiveness of different seeding strategies.

\subsection{Evaluating network seeding strategies}

To assess the effects of seeding, we first tested which of the four seeding strategies, described in Section 2.3, were most effective in generating new adopters over the baseline model of no seeding described in the previous section. We simulated the Random, Highest Degree, Dense Regions, and Hubs Unlikely to adopt seeding strategies over the Yahoo network and varied the fraction of the population targeted to see which produced the greatest increase in product adoption over the baseline. The results are shown in Figure 3, which plots the fraction of the population that adopts the product under each seeding strategy (the $y$-axis) against the fraction of the population that is seeded by a given strategy (the $x$-axis). Several interesting insights emerge.

First, seeding works to some extent. Each of the seeding strategies outperforms the case of no seeding, which is displayed as a relative constant baseline at $0 \%$. 


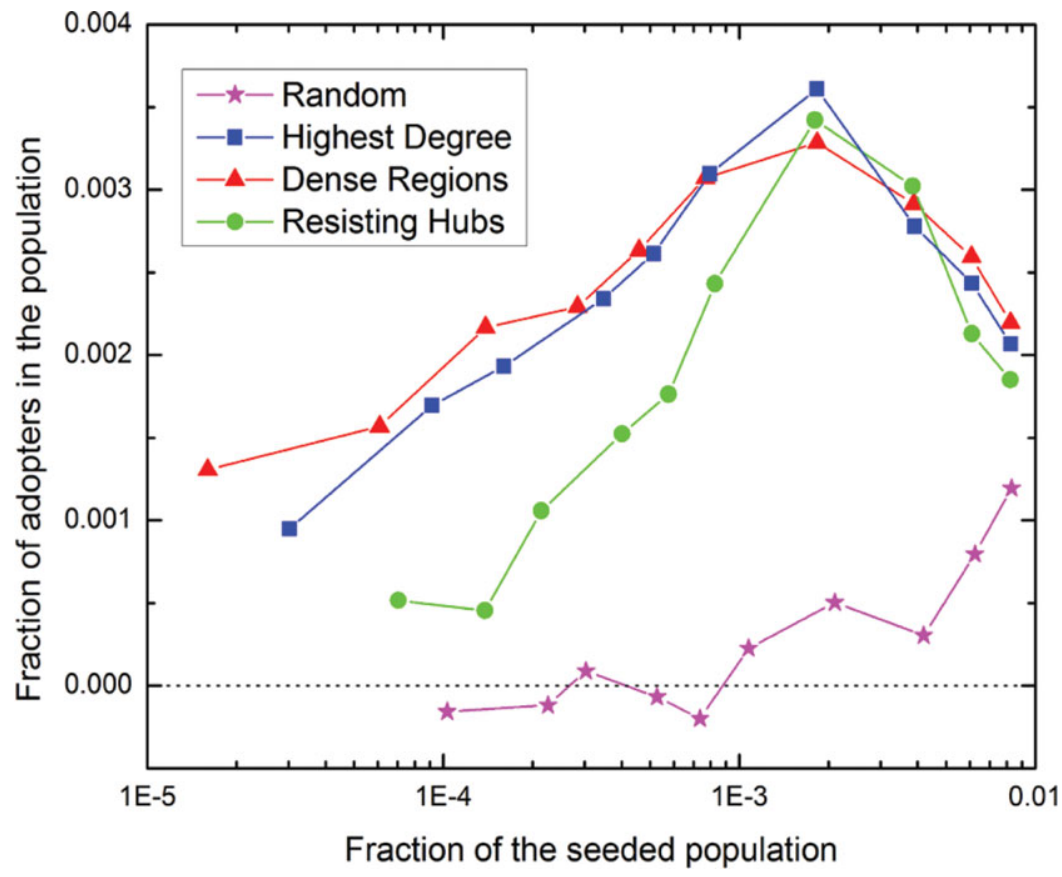

Fig. 3. The relative performance of the tested seeding strategies relative to "no intervention." The figure shows the fraction of the population that adopts the product due to application of various degrees of seeding ( $x$-axis). Lines represent different strategies as defined in the text: random (cyan), highest degree (red), dense regions (green), and resisting hubs (blue). The baseline represents adoption without intentional seeding. (color online)

However, the effect of seeding is small. The most effective seeding strategy achieves at most a $0.4 \%$ increase in product adoption in the population even when the optimal number of targets is selected. When compared to approximately $20 \%$ of the entire population that adopts the product due to individual characteristics and an organic level of peer influence during the same time period, the effect of seeding is underwhelming. As we discuss below, there are several reasons why seeding has an underwhelming effect. Most importantly, only a small fraction of the population is influential enough to be worth seeding.

Second, the three more sophisticated strategies (Highest Degree, Dense Regions, and Hubs Unlikely to Adopt), all outperform random seeding and produce relatively similar results, with Highest Degree and Dense Regions performing the best. This result corroborates previous work on the importance of degree in choosing targets and reiterates the lack of benefit from more complex seeding strategies.

Third, there are diminishing and then negative returns to seeding more than the optimal number of people. In other words, after a certain point, seeding more people in the network reduces the fraction of the paying population that adopts the product. All tested strategies exhibit clear performance maxima when approximately $0.2 \%$ of the highly ranked individuals are targeted. We conducted additional analysis to explore this result and better understand why there are such scope limits to effective seeding. We first tested whether different assumptions about the influence mechanisms at work in the population affect the results. Perhaps if persuasion is 


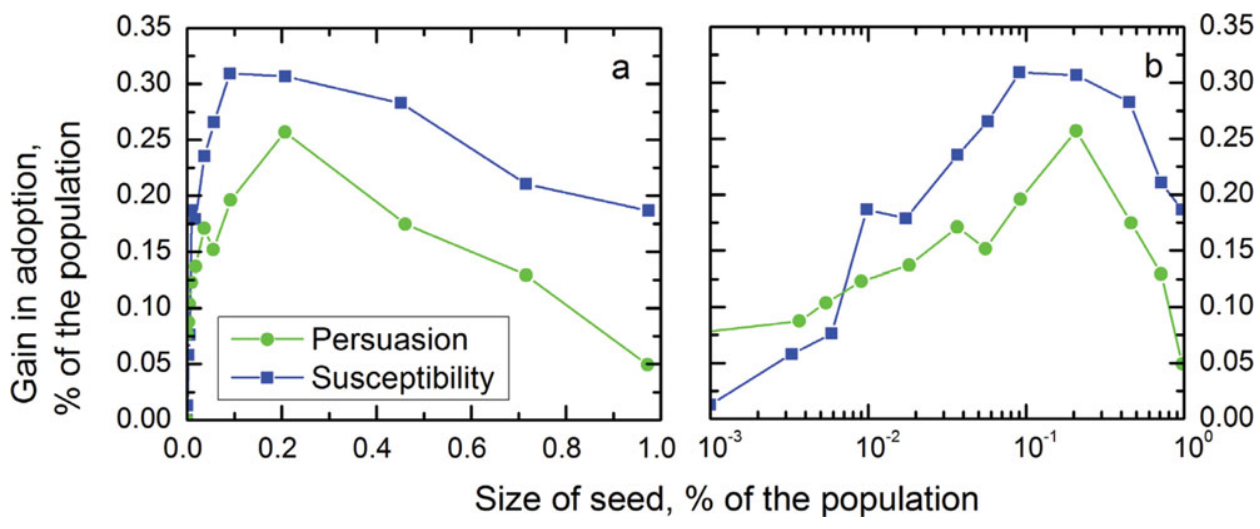

Fig. 4. Panels a \& b show the gain in adoption for persuasion (green) and susceptibility (blue) peer influence mechanisms that are tested for a variety of seeded populations ranging from $0 \%$ to $1 \%$ of the entire population. Panel b presents the data on semi-logarithmic scale to provide sufficient resolution for lower values. (color online)

the predominant force in driving cascades of behavior, seeding is more effective and is optimal at a greater number of targets. To test this explanation, we varied the fraction of the population that was targeted under highest degree seeding (the most effective seeding strategy) with $\gamma=1$ (shown in blue in Figure 4) as well as with $\gamma=0$ (shown in green in Figure 4). The first case represents the scenario in which persuasion is the dominant factor in influence, while the second case represents the scenario in which susceptibility to influence is the dominant factor. Although seeding is indeed more effective when persuasion is more important, under both extreme cases the performance of seeding is greatest when between $0.1 \%$ and $0.2 \%$ of the population are targeted.

While this comparison demonstrates that it is optimal to seed a very small fraction of the population no matter what assumptions are made about the mechanism driving behavioral contagion, it does not explain why the optimal scope of seeding is limited and so small. We measured the expected return on a single target by measuring the average number of additional adopters gained from seeding while varying the fraction of the population targeted (Figure 5(a)). We found that the average gain from seeding drops almost immediately after the best targets are successfully converted. As the plot in Figure 5(a) shows, under both the persuasion regime and the susceptibility regime, there are simply not that many valuable targets in the network. This figure plots the distribution of gross potential influence per consumer, calculated according to our assumptions about how persuasion operates and taking into account network structure, the actual distribution of individual characteristics and adoption propensities in the population, and the excitementbased mechanism of influence (i.e. persuasion is proportional to satisfaction, which is in turn proportional to one's likelihood to adopt the product estimated using individual characteristics). Gross influence is calculated here assuming $\gamma=1$ such that persuasion is maximized:

$$
\text { Gross Influence }_{i}=\sum_{j} w_{i j} \alpha P_{i}^{C H} \text {. }
$$


(a)

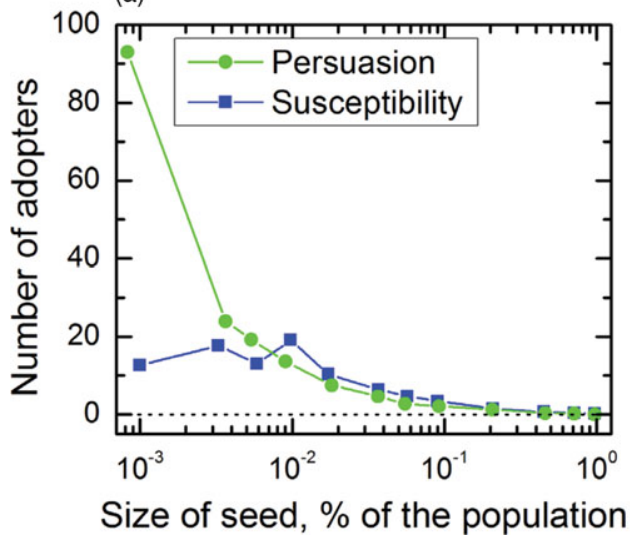

(b)

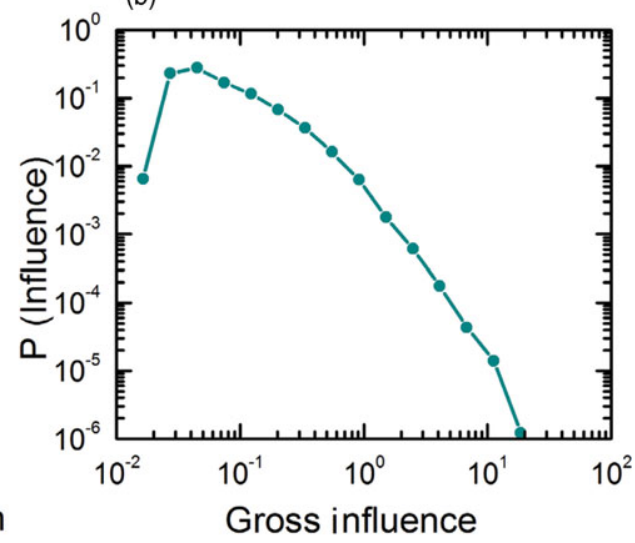

Fig. 5. (a) The average number of adopters generated by a single seed node; (b) a doublelogarithmic plot of the probability density function describing the probability of a user to generate certain amount of influence. (color online)

The distribution of empirically estimated gross potential influence per person in the network is heavy-tailed, meaning there are very few highly influential people to target. This explains why, in our simulations, the effect of seeding decreases so rapidly after seeding the top $0.2 \%$ of influential users. As the plot shows on logarithmic scale, conversion of the most influential consumers is effective and typically results in dozens of secondary adoptions. However, influential consumers, who generate many additional adoptions when they adopt, are extremely rare.

In our simulations, targets who generate on average 10 to 20 additional adopters are approximately 1 in 10,000. These highly influential consumers are followed by moderately influential consumers (those that generate 2 to 5 additional adopters after being targeted) who are more numerous but still only represent 1 in 1,000 consumers. The rest of the consumers in the population are not worth seeding. They are simply not influential enough. They generate less than one additional adopter on average, costing more to target them than their return in additional adoptions. Assuming that the price of conversion is comparable to the gain from adoption, and that the targeted users could have adopted spontaneously, it is not financially beneficial to target this population. Figure 5(a) shows why the effectiveness of seeding strategies is capped at $0.4 \%$ and why we observe negligible variation between different seeding strategies. The number of sufficiently influential individuals is very small and they are quite distinct from the rest of the population, making them easy to identify using any sensible (non-random) seeding policy.

Finally, we tested the greatest overall effect of the most successful seeding scheme and plotted adopters per day compared to natural adoption and natural adoption plus the organic observed level of influence as in Figure 2(b) (see Figure 6). We find almost no perceivable effect of seeding. The number of adopters per day under optimal seeding is almost identical to the case of no seeding.

In essence, it is worth seeding the very few influential users that exist in the network - up to $0.2 \%$ of the population or in our case approximately 54,000 people out of 27 million. However, the overall effect on adoption is small. Depending on the cost of identifying these targets, it may not make sense to target at all. However, 


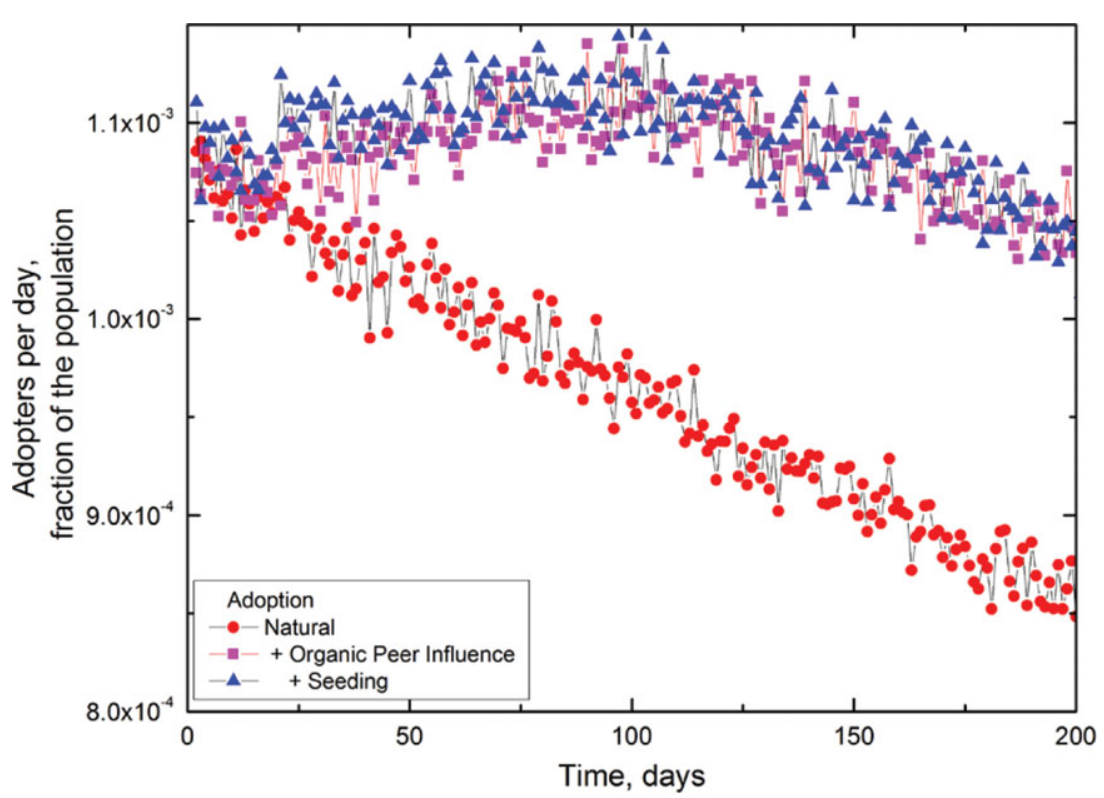

Fig. 6. The simulated daily fraction of adopters under natural (spontaneous) adoption (red), natural adoption + organic peer influence (magenta), $\kappa=0.5$ and natural adoption + organic peer influence + seeding of the product with $0.4 \%$ of the population (blue). The simulation settings are identical to the ones used to generate Figure 2(b). (color online)

since highest degree seeding is relatively easy compared to more complex seeding schemes, such a strategy might still be cost effective.

At this point, it is important to stress the value of data-driven simulation in estimating upper bounds on the effectiveness of firm policies like seeding. A skeptic of simulation studies could argue that these bounds on seeding effectiveness are a function of the assumptions built into our simulation. However, we do not arbitrarily select the parameters in our model. On the contrary, these parameters are empirically estimated in a real consumer network using data on a real product diffusing over this network. Approximately half of the total adoptions of this product in the observed data are spontaneous in which the adopter does not have a prior adopter friend. We also estimate, using dynamic matched sample methods, that at least half of the adoptions that occur with a prior adopter peer in the local network of the adopter are due to individual characteristics or homophily rather than peer influence. Based on these empirical estimates, we bound the amount of organic peer influence that exists in the system and tune the parameters in our simulation to reproduce this amount of organic peer influence. Our validation of the baseline simulation reproduces the original marginal adoption curve quite accurately (see Figure 1). Given this empirical calibration of the simulation parameters, we then test seeding by simply asking what would have happened had the firm converted certain key individuals. We then let the empirically estimated influence parameters simulate subsequent adoptions in the network. In fact, we assess the results over a range of parameters that are reasonable (an order of magnitude above and below our empirical parameter estimates) and confirm that the expected effect of seeding is small and that the optimal target population does not exceed $0.2 \%$ of the consumer 

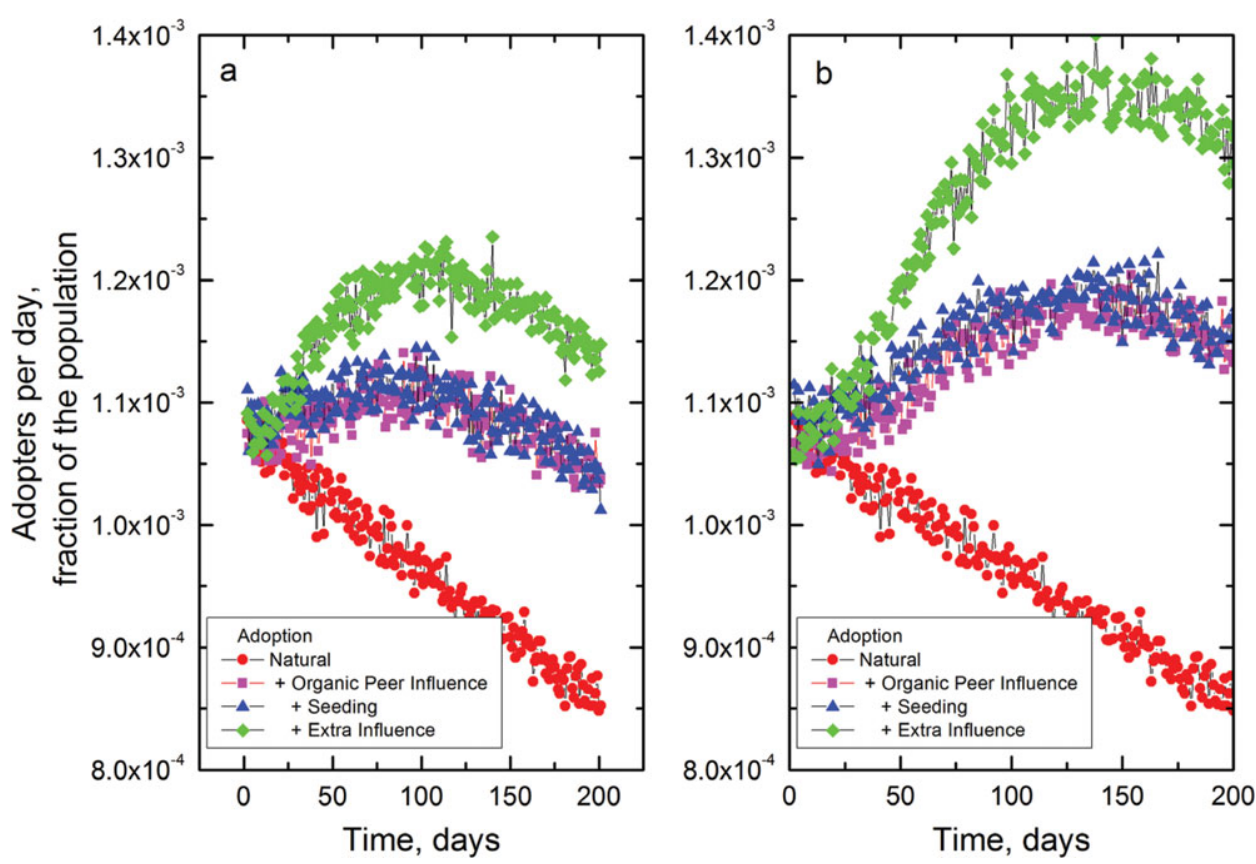

Fig. 7. Daily adoption curves for (a) persuasion $(\gamma=0)$ and (b) susceptibility $(\gamma=1)$. (color online)

population. This gives us confidence that our results are a good representation of what a firm engaged in seeding may expect from their efforts, rather than an outcome based on arbitrary modeling assumptions.

\subsection{Evaluating seeding under varying influence}

Having tested the effects of seeding under observed levels of influence, we turned our attention to assessing the impact of seeding schemes under varying influence. In particular, we were interested in understanding the effects of (a) greater overall influence $(\kappa$ increasing), (b) greater persuasion ( $\gamma$ increasing), and (c) greater susceptibility ( $\gamma$ decreasing) (Aral \& Walker, 2012). We simulated these social conditions by varying $\kappa$ and $\gamma$. As we increased $\kappa$, the overall influence in the system increased. As we increased $\gamma$, adopters were more likely to positively influence the adoption probabilities of their neighbors. As we decreased $\gamma$, peers of adopters were more susceptible to influence and thus more likely to adopt the product once their peers adopted. Manipulation of $\kappa$ represents increases in overall influence due to the characteristics of the product, the social environment, and potentially firm interventions that create incentives to generate greater WOM. Manipulations of $\gamma$ represent varying assumptions about whether influence or susceptibility drive WOMbased adoption and firm incentives being directed at generating positive WOM or susceptibility to peer influence respectively. Our results show dramatic increases in the number of adopters per day as a function of increasing influence (see Figure 7(a) and (b)).

Both persuasion and susceptibility have an effect, though changes in susceptibility have a greater effect on adoption. As shown in Figure 8, a social system in which 


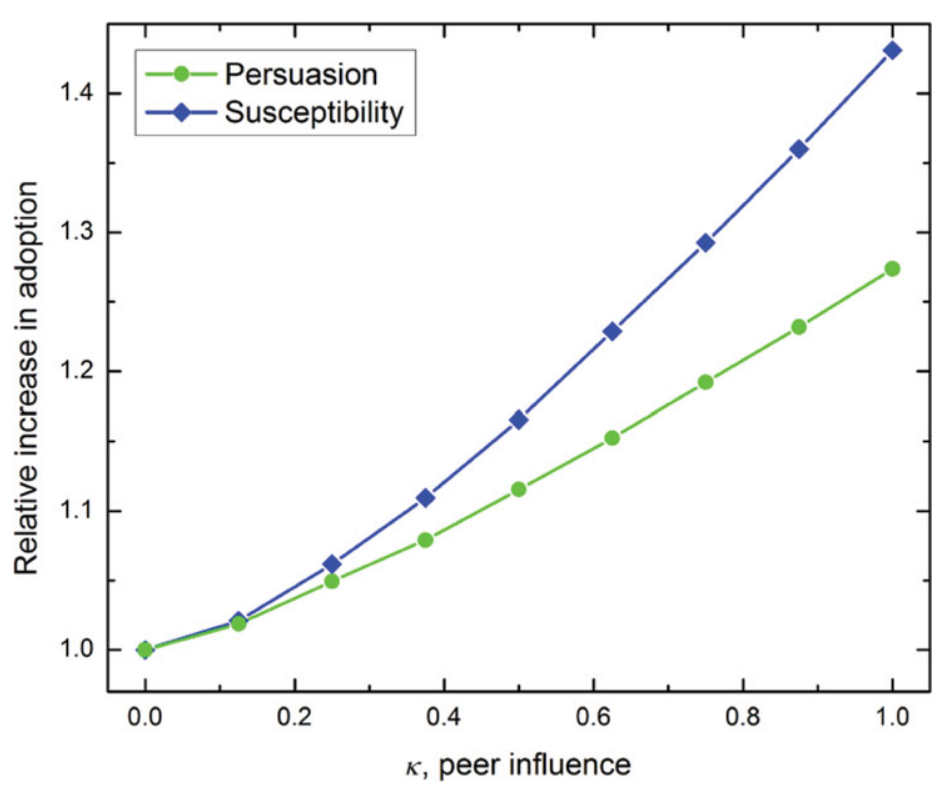

Fig. 8. Relative increase in the number of adopters due to increased peer influence for the peer influence mechanism based on persuasion correlated to the satisfaction from the product (green) and susceptibility (blue). (color online)

only susceptibility to influence drives WOM-based adoption $(\gamma=1$; shown in blue) generates greater increases in adoption than a social system in which only persuasion drives WOM-based adoption ( $\gamma=0$; shown in green), as the overall level of influence in the system is increased ( $\kappa$ increasing). As our prior work estimates that naturally occurring influence calibrates $\kappa$ to be roughly equal to 0.50 , even modest increases in influence above and beyond that amount create significant increase in adoption, with every $10 \%$ increase in influence generating a $5 \%$ increase in total adoption.

For seeding to be successful, targeted consumers must exert some influence on their peers. If there is no increase in peer adoption from converting a target, then seeding will be wasteful in all cases. Extending this logic, as the amount of peer influence in the system increases, seeding should become more effective. This suggests a complementarity between seeding and influence.

We tested this complementarity by fixing a given seeding scheme and simulating adoption while varying the level of peer influence in the system. We simulated complementarity for all of the seeding schemes with similar results, though here we only show results based on the simplest and most effective seeding strategy-highest degree. We varied $\kappa$ under both a pure susceptibility regime $(\gamma=1)$ and a pure persuasion regime $(\gamma=0)$ and measured the relative effect of seeding (compared to the baseline of no seeding) as $\kappa$ increased.

As the results in Figure 9 demonstrate, seeding is clearly more effective under greater levels of influence. When $\kappa$ is set to 0 (far left side of the $x$-axis), seeding has a negative effect because the firm is subsidizing the adoption of some consumers who would have adopted spontaneously had there been no subsidy, while receiving no benefit from their adoption in terms of increases in the adoption probabilities of their peers. However, as influence is increased (moving right along the $x$-axis), seeding 


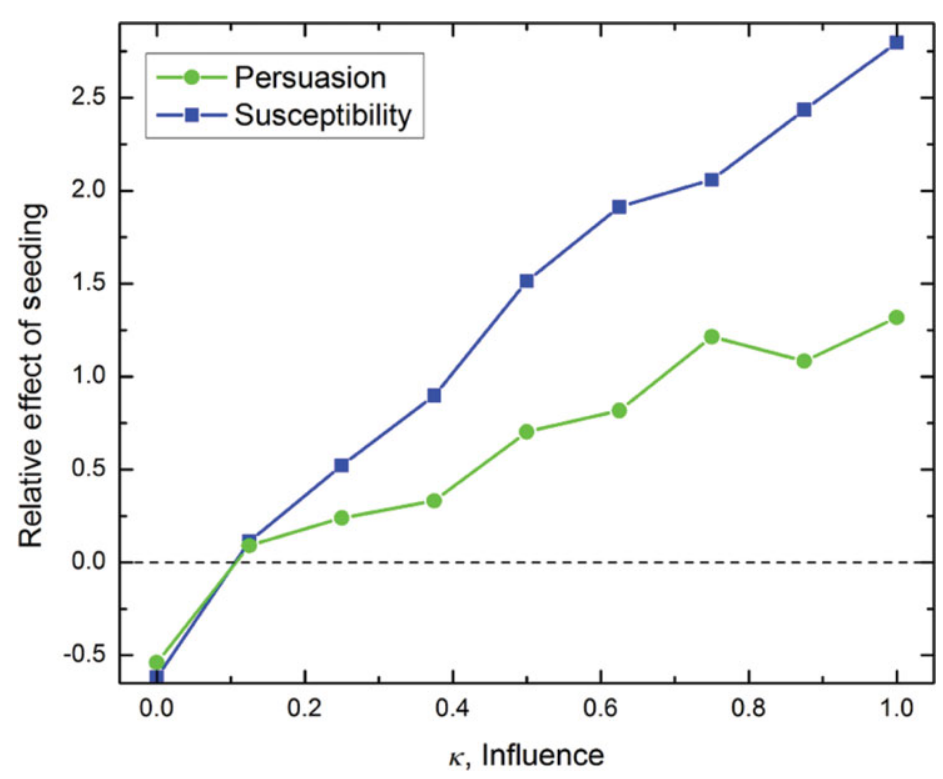

Fig. 9. Cumulative effect of seeding for a range of peer influence effects for susceptibilitydriven (blue) and persuasion-based (green) influence mechanisms. The plot shows the ratio between the number of adopters resulting from adoption with seeding of $0.5 \%$ of the highly connected nodes and the number of adopters in the same scenario without seeding. A slight negative return for seeding with spontaneous adoption represents absence of affect collaborated with the waste of potential adopters due to seeding. (color online)

has a greater effect relative to the baseline of no seeding. At $\kappa=0.25$, seeding has a small but measurable effect on adoption over the baseline, corroborating earlier results of the effects of seeding. Then there are increasing returns as influence is increased. Interestingly, the marginal increase in the return of seeding is diminishing as peer influence grows. This is especially true under greater persuasion (green), for which the positive effects of seeding diminish faster than under greater susceptibility (blue). The variation in the complementarity between seeding and influence under the persuasion and susceptibility regimes was intriguing. We essentially found that seeding is more effective in the presence of influence, but that this complementarity diminishes faster if influence is operating through persuasion than if influence is operating through susceptibility. We investigated further to determine why this was the case.

To guide our exploration, we turned to a result concerning the rate at which hubs adopt in the presence of peer influence. High connectivity exposes highly connected nodes to the influence of early adopters faster (Becker, 1970; Goldenberg et al., 2009). We observe this in our data as well (Figure 10). The average degree of adopters is low and constant over time if influence is set to zero and only spontaneous characteristics-based adoption is considered $(\kappa=0)$. However, the introduction of peer influence dramatically changes the average degree of adopters over time. This indicates that peer influence processes accelerate the adoption of highly connected nodes in our data - a result that is in line with previous work (Becker, 1970; Goldenberg et al., 2009). The acceleration of hubs' adoption is more pronounced for higher levels of influence. But, perhaps more interestingly, hubs adopt faster under 

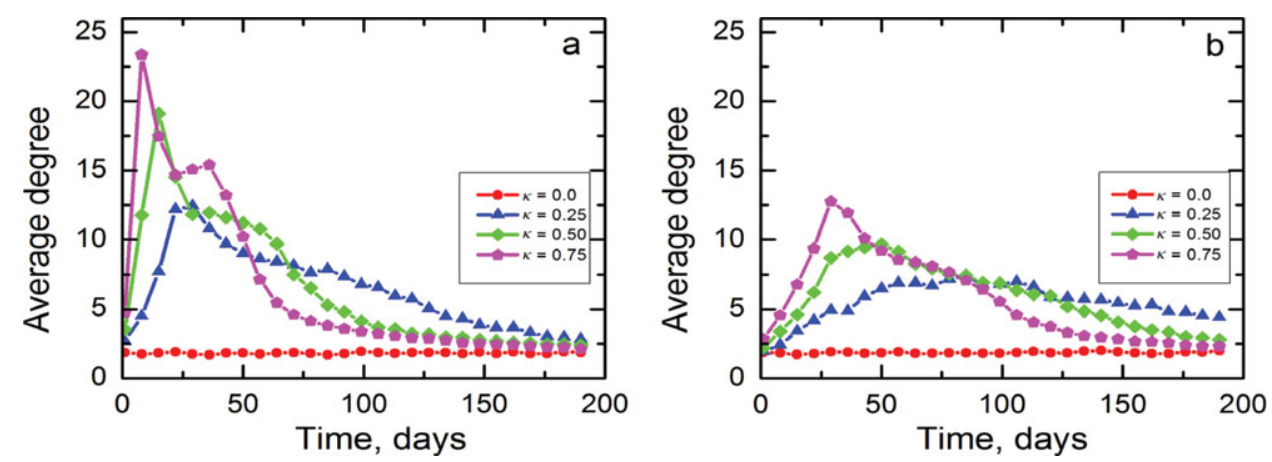

Fig. 10. The average degree of adopters over time for persuasion (left) and susceptibility (right) influence mechanisms. The plots represent different amounts of peer influence in the system: spontaneous adoption (red), natural level of influence (blue), and two incentives campaigns corresponding to double (green) and triple (magenta) the natural influence. (color online)

increases in persuasion than under increases in susceptibility, so there is less of a need for the firm to seed them under a persuasion regime than under a susceptibility regime (see Figure 10). These results explain why the complementarity between seeding and incentives diminishes faster under the persuasion regime than under the susceptibility regime.

\subsection{The effect of homophily}

The benefit of data-driven simulation is that modeling assumptions are based on realistic, empirically estimated model parameters. The likelihood of adoption as a function of individual characteristics and the amount of peer influence in the adoption process are based on empirically observed values. One of the most important dimensions of adoption diffusion that must be estimated and modeled realistically is homophily or assortativity. How different types of consumers are distributed over the network can have a dramatic impact on the diffusion process and the likely effects of seeding (Aral et al., 2009; McPherson et al., 2001; Noel \& Nyhan, 2011; Shalizi \& Thomas, 2011). We therefore tested how homophily affects our results.

We tested our simulations under three different assumptions about the amount of homophily or assortativity in the network. The first uses the observed distributions of individual characteristics over the nodes in the network (Original). This formulation preserves the original amount of homophily in the network that connects consumers. The second uses a shuffled graph in which we randomly shuffled the observed characteristics of individuals in the graph maintaining the distributions of observed characteristics in the data (Randomly Shuffled). This makes the distribution of characteristics over the graph completely random while preserving the distributions of these characteristics in the population. The third uses homogeneous characteristics, in which all consumers display the average value for each characteristic (homogeneous). These three variations in assortativity affect the correlations in the likelihood of adoption amongst linked nodes and thus change the degree to which likely adopters are or are not clustered in the network. We use simulations under 

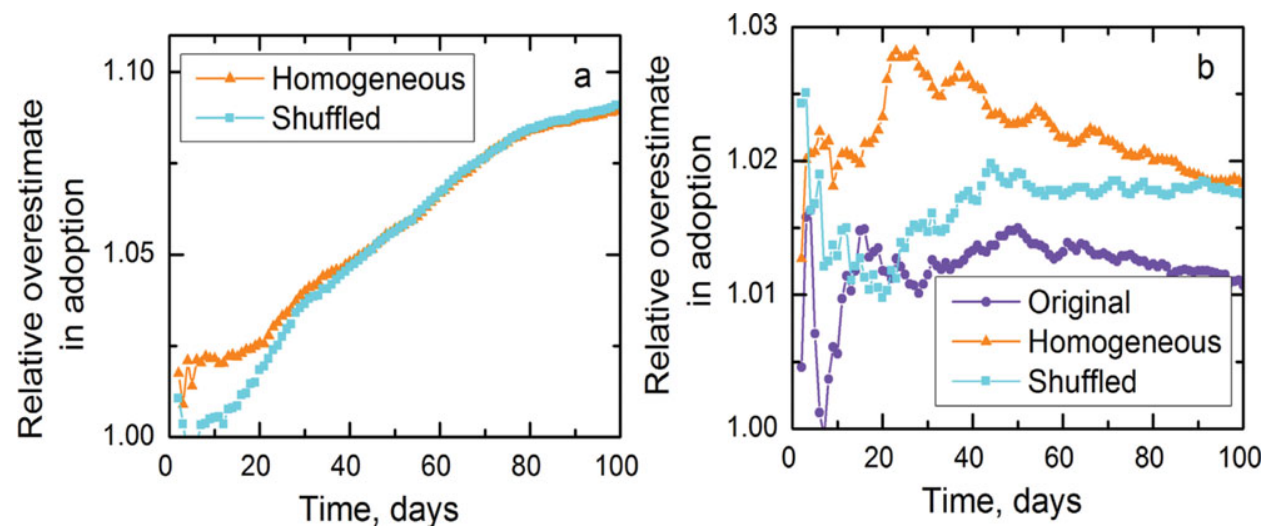

Fig. 11. (a) The ratio between the numbers of adopters in homogeneous (orange) and shuffled (cyan) cases versus the number of adopters estimated using the real observed amount of homophily in the network. These simulations correspond to $\kappa=0.5$ and $\gamma=0$; (b) the effectiveness of seeding by plotting the ratio between the cumulative number of adopters for the case when $0.2 \%$ of the most connected users were seeded and the case were no seeding was performed. The plots correspond to the three tested distributions of user characteristics in the network: original (red), shuffled (green), and homogeneous (blue). (color online)

these three states to test whether our results are sensitive to realistic assumptions about the degree of homophily in the network.

Figure 11 compares estimates of the number of adopters under shuffled characteristics and homogeneous characteristics to the same estimates under the original amount of homophily in the observed network. The artificial cases represent typical approaches in most simulation studies of network seeding and WOM which ignore homophily. The results show consistent and dramatic overestimates of the cumulative adoption in the network in both the shuffled and homogeneous cases over time. These overestimates are approximately the same as we vary the influence mechanism in the model (persuasion and susceptibility). The degree of overestimation increases over time as the contagion effect propagates through the network and reaches approximately a $10 \%$ overestimate after just 100 days from the launch of the product (see Figure 11(a)).

While the overestimate seems moderate, the raw results are misleading. Since most users adopt the product spontaneously without influence and since contagion is responsible for only about $20 \%$ of adoptions in this setup, failure to realistically model homophily leads to a $30 \%$ overestimate of contagion in the network in the first 100 days. As the simulation continues over time, these overestimates continue to increase, though at a diminishing rate. Furthermore, the fact that the overestimates are nearly identical for the shuffled and homogeneous cases suggests that merely considering the right distribution of characteristics in the consumer population, rather than homophily itself, leads to similarly misguided expectations of contagion. These results are dramatic and call into question simulations of network propagation that do not employ realistic assumptions about homophily and assortativity.

Next we tested whether ignoring realistic degrees of homophily affects estimates of the effectiveness of seeding strategies. We again tested the effect of seeding $0.2 \%$ of the most connected users in the three cases - the original, shuffled, and 

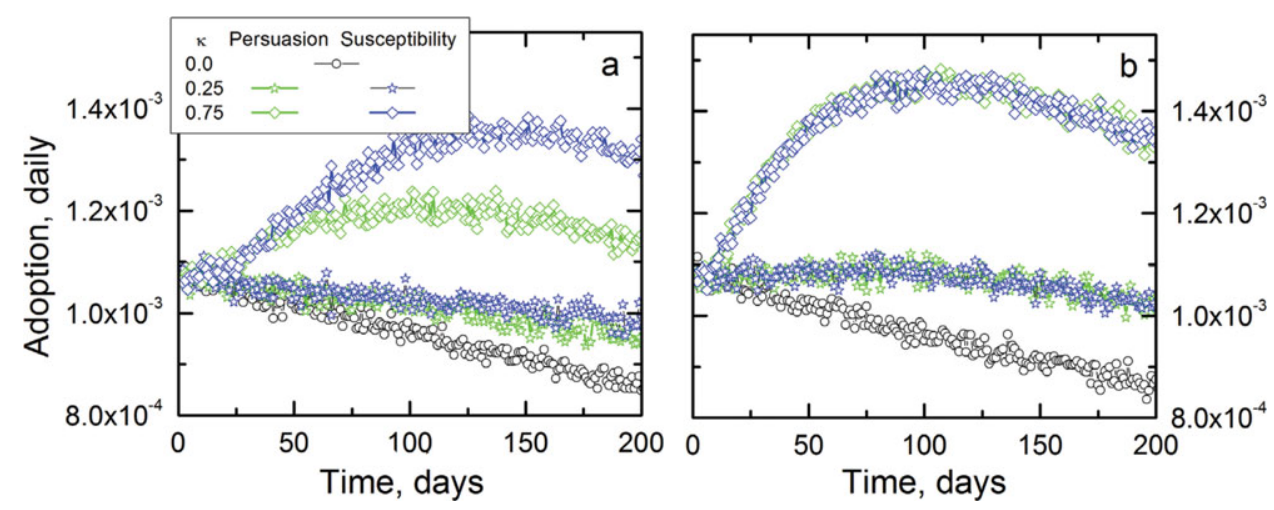

Fig. 12. (a) Daily adoption rate is computed for five peer influence models: spontaneous adoption with no influence present (black circles), natural level of peer influence according to the satisfaction-driven model of influence with $\kappa=0.25, \gamma=0$ (green stars), natural level of influence according to the model of susceptibility-driven influence with $\kappa=0.25, \gamma=1$ (blue stars), and two corresponding scenarios with enhanced peer influence for $\kappa=0.75$ and $\gamma=0$ (greens diamonds) and $\gamma=1$ (blue diamonds). (b) The figure shows daily adoption rate for the same set of scenarios as in panel (a) but for the shuffled network (see the paper text for details). (color online)

homogeneous networks (Figure 11(b)). The plots confirm that the effect of seeding is also overestimated when homophily is ignored. The overestimate varies over time and depends on the strength of peer influence in the system. In the typical setup, disregarding homophily leads to a $200 \%$ overestimate of the effect of seeding.

Finally, we tested the effects of ignoring homophily under different assumptions about the degree of influence in the system. Figure 12 plots simulated daily adoption rates varying the amount and types of influence in the system. Figure 12(a) reproduces the original graph in Figure 7 for comparison and Figure 12(b) simulates the same assumptions and policies for a network in which individual characteristics are randomly shuffled. The results are dramatic. As is clear from the Figure, when homophily is ignored, persuasion and susceptibility regimes create exactly the same effect on the number of adopters per day (Figure 12(a)). However, when the real assortativity is considered, a system in which susceptibility dominates creates noticeably more adoption than a system in which persuasion dominates (Figure 12(a)).

The difference in adoption between the two influence regimes is caused by the interplay between the distribution of user traits in the network and the way different populations of users are favored by each of the two regimes. In the persuasion regime, the influence applied by adopter $i$ on her neighbors is the same for every neighbor (i.e. $P_{i t}^{I}=\kappa \sum_{j \in N_{i}} w_{i j t} y_{j t-1}\left(P_{i t}^{C H}\right)$ ). In the susceptibility regime, influence is selective: adopters' neighbors experience influence that is proportional to their own $P_{j t}^{C H}$. While in the first case influence is dispersed equally between all adopter friends, in the second case higher influence is experienced by those who are more likely to adopt spontaneously. Even though on average the adoption propensity of the initial adopter $\left(P_{i t}^{C H}\right)$ is higher than the adoption propensity of her peers $\left(P_{j t}^{C H}\right)$, some peers have a significantly higher propensity to adopt than that of their adopter friends. In the susceptibility regime, influence is applied disproportionally to these adopters who are themselves more likely to adopt. 

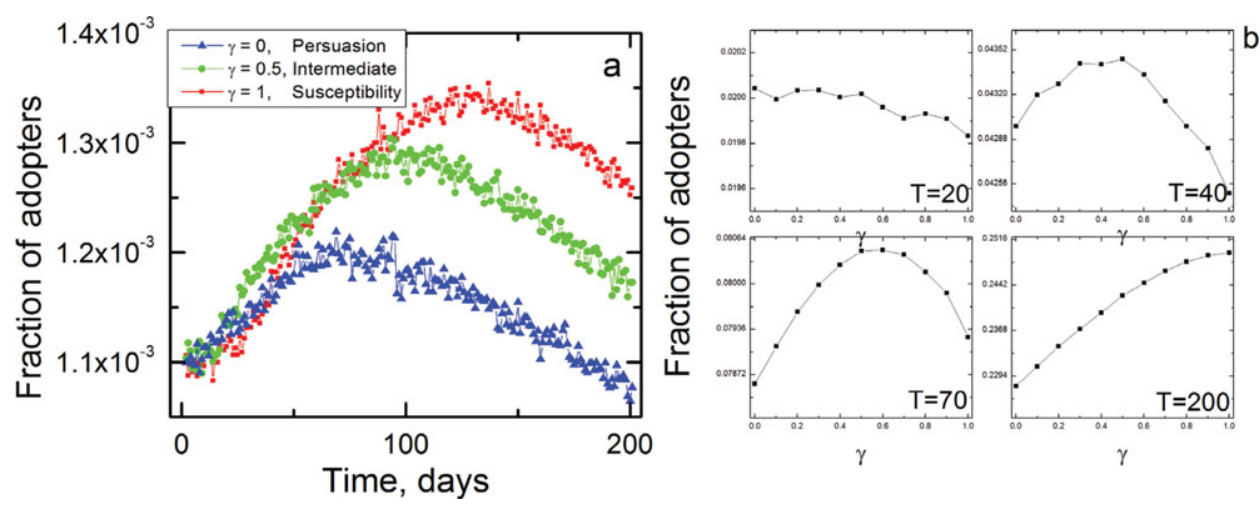

Fig. 13. (a) The adoption curves corresponding to persuasion (red), mixed (green), and susceptibility (blue) models of peer influence; (b) the dependence of the fraction of adopters in the populations on $\gamma$ for four time slices. (color online)

The susceptibility-driven influence process therefore selectively diffuses through the network toward clusters of individuals with a greater likelihood of adoption. When a natural level of homophily is appropriately modeled, adopters tend to be surrounded by potential adopter peers with higher $P_{j t}^{C H}$. Due to homophily, influence is routed toward areas of the network populated with more likely adopters in the susceptibility model, creating higher levels of overall influence. Random shuffling of $P^{C H}$ across the network eliminates naturally occurring homophily. Consequently, the correlation of $P^{C H}$ between peers disappears and the selection of users more likely to adopt does not divert influence toward areas of the network which contain the most likely adopters. This creates nearly identical adoption diffusion dynamics across the persuasion and susceptibility regimes when homophily is ignored.

To see the relative effects in persuasion and susceptibility regimes, Figure 13 plots simulated daily adoption rates varying the types of influence in the system. All simulations produce nearly identical rates of adoption in the first 50 days from product launch since, regardless of the peer influence model used, no influence exists in the system as there are few adopters to generate it. However, differences in estimates of adoption between persuasion and susceptibility regimes clearly develop over time. As the product diffuses, the susceptibility regime generates more adoption than the persuasion regime (Figure 13(a)). Figure 13(b) shows that in the early days of the diffusion process ( day 20), persuasion $(\gamma=0)$ is slightly more diffusive than susceptibility $(\gamma=1)$. However, as time progresses, the $\gamma$ that generates the most adoption moves closer to 1 until, at approximately the 200th day, the pure susceptibility regime creates the most adoption. As the diffusion unfolds, the susceptibility regime creates more adoption in the network over time as the selection process drives influence toward clusters of individuals who are more likely to adopt.

In summary, when behaviors and homophily are modeled realistically, the persuasion and susceptibility regimes exhibit distinct product diffusion patterns. Increases in peer influence created by persuasion cause wider exposure to the product, but increases in susceptibility of adopters' peers to influence cause a selective exposure to those peers who have a natural inclination to adopt the product, creating more peer adoption in the network. 


\section{Discussion: Policy implications, limitations, and future work}

We conducted realistic data-driven simulations of social contagion in product adoption and tested different network seeding strategies under different social conditions. Our results highlight the importance of using real-world data to guide modeling assumptions and challenge conventional wisdom about the likely effects of seeding in several ways.

First, most seeding strategies exhibit relatively similar performance. Although we tested several different seeding strategies discussed in the literature, our results show that they are approximately indistinguishable in their effect on product adoption. Seeding the highest degree nodes performs as well if not better than more sophisticated seeding strategies. As identifying influentials based on their degree is relatively easy and less costly for the firm because only degree distributions are needed (rather than more complex network variables), these results indicate that firms need not invest in more sophisticated strategies that rely on identifying influentials with more complex network data.

Second, seeding a small fraction of the most influential consumers in the population pays off as there are returns to seeding the most influential nodes. However, the scope of optimal seeding policies is limited. Seeding greater than $0.2 \%$ of the population is wasteful because the gains in adoptions influenced by seeding more consumers are smaller than the gains from their organic adoption after $0.2 \%$ of the most influential consumers have been seeded. These results suggest that firms need not invest in large seeding programs, but rather in seeding policies of limited scope that focus only on the most influential individuals (in our case identified by the highest degree). These results hold for all the seeding strategies we tested including highest degree and the more sophisticated strategies.

Third, seeding is more effective in the presence of greater influence. Interestingly, there are diminishing marginal complementarities between seeding and influence as influence is increased. The marginal increase in the return of seeding is diminishing as peer influence grows and is diminishing faster when persuasion drives influence than when susceptibility drives influence. This is because hubs adopt faster under increases in persuasion than increases in susceptibility, so there is less of a need for the firm to seed them under a persuasion regime than under a susceptibility regime.

Finally, ignoring homophily creates significant overestimates of seeding strategies - up to $30 \%$. Ignoring homophily also changes the interpretation of which regime, persuasion or susceptibility, creates more adoption. In the susceptibility regime, influence is applied disproportionally to adopters who are themselves more likely to adopt. When homophily is appropriately accounted for in the model, the susceptibility-driven influence process selectively diffuses through the network toward clusters of individuals with a greater likelihood of adoption, creating higher levels of overall influence.

There are some limitations to our work, caveats about how the results should be interpreted and obvious directions for future research. First, although we use a large dynamic networked dataset and a real product diffusing over the same network to estimate our model parameters, we (like all simulation studies) assume seeding is "successful" at some baseline level. In other words, we assume that seeded nodes adopt the product. We do not make any claims about the local effects of each 
of these strategies (e.g. how successful seeding strategies are at converting targeted consumers). Our goal is instead to assume these efforts are successful and then to model the likely global diffusion effects of such strategies. There is some evidence that seeding is successful at converting targeted users (e.g. Iyengar et al., 2011). As cost considerations are not essential to our models, we think these baseline assumptions are reasonable. However, future work should explore how investment is related to success and for example whether investment increases marketing effectiveness at diminishing rates.

Second, future work should consider more complex and comprehensive models of peer influence. For example, we did not consider the effects of making peer influence a function of the similarity between peers or local network properties such as triadic closure or the number of mutual friends two consumers have in common. In addition, although our model fit our data well, little is known in general about the most appropriate functional form of influence models or whether they generalize across different contexts. The generalizability of the findings needs to be further explored given that the empirical evidence comes from a single setting. We adopted a parsimonious approach to simplify the model while preserving its essential characteristics and model fit. We see potential in future work that tests the robustness of the model further. For example, collecting data from several examples of the diffusion of products over networks could help test our specification in multiple other settings. We are however also acutely aware of the difficulty of obtaining data like those we have presented.

Third, our model of adoption is calibrated with parameters that are fixed in time. The model is trained on the adoption data from the first 40 days of the adoption process and simulations use these estimated model parameters over the longer time horizons. In reality, propensities to adopt the product due to individual characteristics and peer influence may change over time and over the product's life cycle. Future work should consider how dynamic estimation of model parameters changes the results.

Finally, we only considered varying amounts of positive WOM. In all likelihood, negative WOM and churn also affect adoption and diffusion dynamics. As relatively few studies examine negative WOM with some notable exceptions (e.g. Singh, 1990), we encourage more work on its effect on contagion and product diffusion.

\section{Conclusion}

We used data on a real, large-scale social network of 27 million individuals interacting daily together with the day-by-day adoption of a new mobile service product, to inform, build, and analyze a data-driven simulation of the effectiveness of seeding strategies under different social conditions. Three main results emerge from our simulations. First, failure to consider homophily, as the bulk of current literature does, creates significant overestimation of the effectiveness of seeding strategies, casting doubt on conclusions drawn by simulation studies that do not model homophily. Second, in addition to generating relatively fewer total adoptions, seeding is constrained by the relatively small fraction of potential influencers that exist in the network. We find that seeding more than $0.2 \%$ of the population 
is wasteful because the gain from their adoption is lower than the gain from their natural adoption (without seeding). Third, seeding is more effective under greater social influence. Our findings call into question some of the conventional wisdom about seeding strategies and suggest their overall effectiveness may be overestimated.

\section{Supplementary materials}

For supplementary material for this article, please visit http://dx.doi.org/10.1557/ nws.2012.3.

\section{References}

Abrahamson, E., \& Rosenkopf, L. (1997). Social network effects on the extent of innovation diffusion: A computer simulation. Organization Science, 8(3), 289-309.

Aral, S. (2011). Identifying social influence: A comment on opinion leadership and social contagion in new product diffusion. Marketing Science, 30(2), 217-223.

Aral, S., Muchnik, L., \& Sundararajan, A. (2009). Distinguishing influence-based contagion from homophily-driven diffusion in dynamic networks. Proceedings of the National Academy of Sciences of the United States of America, 106(51), 21544-21549. doi:10.1073/pnas.0908800106

Aral, S., \& Taylor, S. (2011). Viral incentive systems: A randomized field experiment. Workshop on Information Systems Economics (WISE), December 7-9, Shanghai, China.

Aral, S., \& Van Alstyne, M. (2011). The diversity-bandwidth tradeoff. American Journal of Sociology, 117(1), 90-171.

Aral, S., \& Walker, D. (2011). Creating social contagion through viral product design: A randomized trial of peer influence in networks. Management Science, 57(9), 1623-1639.

Aral, S., \& Walker, D. (2012). Identifying influential and susceptible members of social networks. Science, 337(6092), 337-341. doi:10.1126/science.1215842

Bakshy, E., Eckles, D., Yan, R., \& Rosenn, I. (2012a). Social influence in social advertising: Evidence from field experiments. Proceedings of the 13th ACM Conference on Electronic Commerce (EC), June 8-12, Valencia, Spain.

Bakshy, E., Rosenn, I., Marlow, C., \& Adamic, L. (2012b). The role of social networks in information diffusion. Proceedings of the 21st International Conference on World Wide Web $(W W W)$, April 16-20, Lyon, France.

Barabási, L. (2002). Linked: How Everything is Connected to Everything Else and What it Means for Business, Science and Everyday Life. New York: Penguin Group.

Barthélemy, M., Barrat, A., Pastor-Satorras, R., \& Vespignani, A. (2004). Velocity and hierarchical spread of epidemic outbreaks in scale-free networks. Physical Review Letters, 92(17), 178701. doi:10.1103/PhysRevLett.92.178701

Bass, F. M. (1969). A new product growth for model consumer durables. Management Science, 15(5), 215-227. doi:10.1287/mnsc.15.5.215

Becker, M. H. (1970). Factors affecting diffusion of innovations among health professionals. American Journal of Public Health, 60(2), 294-304.

Biyalogorsky, E., Gerstner, E., \& Libai, B. (2008). Customer referral management: Optimal reward programs. Marketing Science, 20(1), 82-95.

Borgatti, S. P. (2005). Centrality and network flow. Social Networks, 27(1), 55-71. doi:10.1016/j.socnet.2004.11.008

Buttle, F. A. (1998). Word of mouth: Understanding and managing referral marketing. Journal of Strategic Marketing, 6(3), 241-254. 
Centola, D. (2010). The spread of behavior in an online social network experiment. Science, 329(5996), 1194-1197.

Centola, D. (2011). An experimental study of homophily in the adoption of health behavior. Science, 334(6060), 1269-1272.

Centola, D., \& Macy, M. (2007). Complex contagions and the weakness of long ties. American Journal of Sociology, 113(3), 702-734.

Chatfield, C., \& Goodhardt, G. J. (1973). A consumer purchasing model with erlang interpurchase times. Journal of the American Statistical Association, 68, 828-835.

Christakis, N. A., \& Fowler, J. H. (2007). The spread of obesity in a large social network over 32 years. The New England Journal of Medicine, 357(4), 370-379.

Cohen, R., Erez, K., ben-Avraham, D., \& Havlin, S. (2001). Breakdown of the Internet under intentional attack. Physical Review Letters, 86, 368-3685

Dezső, Z., \& Barabási, A.-L. (2002). Halting viruses in scale-free networks. Physical Review E, 65(5), 055103. doi:10.1103/PhysRevE.65.055103

Domingos, P., \& Richardson, M. (2001). Mining the network value of customers. Paper presented at the International Conference on Knowledge Discovery and Data Mining, San Francisco, California. http://portal.acm.org/citation.cfm?id=502525\# .

Dover, Y., Goldenberg, J., \& Shapira, D. (2012). Network Traces on Penetration: Uncovering Degree Distribution from Adoption Data. Marketing Science, 31(4), 689-712.

Eagle, N., Macy, M., \& Claxton, R. (2010). Network diversity and economic development. Science, 328(5981), 1029-1031.

Freeman, L. C. (1979). Centrality in social networks: Conceptual clarification. Social networks, 1, 215-239.

Galeotti, A., \& Goyal, S. (2009). Influencing the influencers: A theory of strategic diffusion. The RAND Journal of Economics, 40(3), 509-532. doi:10.1111/j.1756-2171.2009.00075.x

Gladwell, M. (2000). Tipping points: How little things can make a big difference. Boston, MA: Little, Brown and Company.

Godes, D., \& Mayzlin, D. (2009). Firm-created word-of-mouth communication: Evidence from a field test. Marketing Science, 28(4), 721-739.

Goldenberg, J., Han, S., Lehmann, D. R., \& Hong, J. W. (2009). The role of hubs in the adoption process. Journal of Marketing, 73(2), 1-13.

Goldenberg, J., Libai, B., \& Muller, E. (2001). Talk of the network: A complex systems look at the underlying process of word-of-mouth. Marketing Letters, 12(3), 211-223.

Goyal, A, Bonchi, F, \& Lakshmanan, L. V. S. (2010). Learning influence probabilities in social networks. Proceedings of the 3rd ACM International Conference on Web search and Data Mining (WSDM), February 4-6, New York, USA.

Iyengar, R., Van den Bulte, C., \& Valente, T. W. (2011). Opinion leadership and social contagion in new product diffusion. Marketing Science, 30(195).

Katz, E., \& Lazarsfeld, P. (1955). Personal influence. New York: Free Press.

Kempe, D., Kleinberg, J., \& Tardos, É. (2003). Maximizing the spread of influence through a social network. Paper presented at the Ninth ACM SIGKDD international conference on Knowledge discovery and data mining, Washington, DC.

Kitsak, M., Gallos, L. K., Havlin, S., Liljeros, F., Muchnik, L., Stanley, H. E., \& Makse, H. A. (2010). Identification of influential spreaders in complex networks. Nature Physics, 6(11), 888-893. doi:10.1038/nphys1746

Krebs, V. E. (2002). Uncloaking terrorist networks. First Monday, 7(4). Retrieved from http://www.firstmonday.dk/issues/issue7_4/krebs/index.html.

Lazarsfeld, P. F., Berelson, B., \& Gaudet, H. (1948). The people's choice. New York: Columbia University Press. 
Lazer, D., Pentland, A., Adamic, L., Aral, S., Barabási, A.-L., Brewer, D.,... Alstyne, M. V. (2009). Computational social science. Science, 323(5915), 721-723. doi:10.1126/ science. 1167742

Leider, S., Mobius, M. M., Rosenblat, T., \& Do, Q.A. (2009). Directed altruism and enforced reciprocity in social networks. Quarterly Journal of Economics, 124(4), 1815-1851.

Leskovec, J., Adamic, L. A., \& Huberman, B. A. (2007). The dynamics of viral marketing. ACM Transactions on the Web, 1(1).

Libai, B., Biyalogorsky, E., \& Gerstner, E. (2003). Setting referral fees in affiliate marketing. Journal of Service Research, 5(4), 303-315. doi:10.1177/1094670503005004003

Libai, B., Muller, E., \& Peres, R. (2009). The Social Value of Word-of-Mouth Programs: Acceleration versus Acquisition. Working Paper No. 22/2009, Recanati Business School, Tel Aviv University.

Liben-Nowell, D., \& Kleinberg., J. (2008). Tracing information flow on a global scale using internet chain-letter data. Proceedings of the National Academy of Sciences of the United States of America, 105(12), 4633-4638.

Marsden, P. (2006). Seed to spread: How seeding trials ignite epidemics of demand. In J. Kirby, \& P. Marsden (Eds.), Connected marketing: The viral, buzz, and word of mouth revolution (pp. 3-23). Oxford: Butterworth-Heinemann.

McPherson, M., Smith-Lovin, L., \& Cook, J. M. (2001). Birds of a feather: Homophily in social networks. Annual Review of Sociology, 27, 415-444. doi:10.1146/annurev.soc.27.1.415

Newman, M. E. J. (2003). The structure and function of complex networks. SIAM Review, 45, $167-256$.

Noel, E., \& Nyhan, B. (2011). The "unfriending" problem: The consequences of homophily in friendship retention for causal estimates of social influence. Social Networks, 33(3), 211-218.

Pastor-Satorras, R., \& Vespignani, A. (2001). A. Epidemic spreading in scale-free networks. Physical Review Letters, 86, 320-3203.

Reingen, P. H., \& Kernan, J. B. (1986). Analysis of referral networks in marketing: Methods and illustration. Journal of Marketing Research, 23(4), 370-378.

Rosen, E. (2009). The anatomy of buzz revisited: Real-life lessons in word of mouth marketing. New York: Random House Digital, Inc.

Rosvall, M., \& Bergstrom, C. T. (2008). Maps of random walks on complex networks reveal community structure. Proceedings of the National Academy of Sciences of the United States of America , 105(4), 1118-1123. doi:10.1073/pnas.0706851105

Schmitt, P., Skiera, B., \& Van den Bulte, C. (2011). Referral programs and customer value. Journal of Marketing, 75(1), 46-59. doi:10.1509/jmkg.75.1.46

Shalizi, C. R., \& Thomas, A. C. (2011). Homophily and contagion are generically confounded in observational social network studies. Sociological Methods Research, 42(2), 211-239.

Singh, J. (1990). Voice, exit, and negative word-of-mouth behaviors: An investigation across three service categories. Journal of the Academy of Marketing Science, 18(1), 1-15. doi:10.1177/009207039001800101

Sun, E., Rosenn, I., Marlow, C., \& Lento, T. (2009). Gesundheit! modeling contagion through Facebook news feed. Paper presented at the Third International Conference on Weblogs and Social Media, San Jose, CA.

Sundararajan, A. (2008). Network seeding. Mimeo: New York University.

Taylor, S., Bakshy, E., \& Aral, S. (2013). Selection Effects in Online Sharing: Consequences for Peer Adoption, 14th ACM Conference on Electronic Commerce (EC), June 16-20, University of Pennsylvania, Philadelphia, PA.

Uncles, M., Ehrenberg, A., \& Hammond, K. (1995). Patterns of buyer behavior: Regularities, models, and extensions. Marketing Science, 14(3), G71-G78. doi:10.1287/mksc.14.3.G71

Valente, T. W. (2012). Network interventions. Science, 337(6090), 49-53. doi:10.1126/ science. 1217330 
Valente, T. W., \& Davis, R. L. (1999). Accelerating the diffusion of innovations using opinion leaders. The ANNALS of the American Academy of Political and Social Science, 556(1), 55-67. doi:10.1177/000271629956600105

Van den Bulte, C., \& Joshi, Y. V. (2007). New product diffusion with influentials and imitators. Marketing Science, 26(3), 400-421. doi:10.1287/mksc.1060.0224

Watts, D. J., \& Dodds, P. S. (2007). Influentials, networks, and public opinion formation. Journal of Consumer Research, 34, 441-458.

Whyte, W. H. (1954). The web of word of mouth. Fortune, 5, 140-212. 